\title{
Noninvasive brain stimulation for dysphagia after acquired brain injury : a systematic review
}

\author{
Ryo Momosaki, Shoji Kinoshita, Wataru Kakuda, Naoki Yamada, and Masahiro Abo \\ Department of Rehabilitation Medicine, The Jikei University School of Medicine, Nishi-Shimbashi, Minato-Ku, Tokyo, Japan
}

\begin{abstract}
The purpose of this study was to review the best available evidence of noninvasive brain stimulation, including repetitive transcranial magnetic stimulation (rTMS) and transcranial direct current stimulation (tDCS) for dysphagia after acquired brain injury. We searched randomized controlled trials that compared noninvasive brain stimulation with control used to improve dysphagia after acquired brain injury. We assessed dysphagia severity rating scales and penetration-aspiration scale as outcomes immediately after intervention. We calculated the pooled estimate of the standardized mean difference (SMD) to combine individual results. We included 8 published studies. Two heterogenous trials of 48 patients showed that rTMS was associated with a significant improvement in the dysphagia severity rating scale score (SMD 2.95). Three homogeneous trials of 88 patients showed a significant effect of rTMS on the penetration-aspiration scale score (SMD 0.77). Two homogeneous trials of 34 patients showed that tDCS was associated with a significant improvement in the dysphagia severity rating scale score (SMD 1.20). The review provided low - quality evidence for the effectiveness of noninvasive brain stimulation in improving dysphagia after acquired brain injury. Further trials of larger sample sizes are needed to determine the most appropriate noninvasive brain stimulation protocol. J. Med. Invest. 63 : 153 - 158, August, 2016
\end{abstract}

Keywords : transcranial magnetic stimulation, transcranial direct current stimulation, deglutition disorders, stroke, meta-analysis

\section{INTRODUCTION}

Acquired brain injury (ABI), including stroke and traumatic brain injury, is a potentially serious condition that frequently results in disability requiring rehabilitation (1). Dysphagia is a common complication in ABI patients and is a major cause of aspiration pneumonia and malnutrition. Dealing with dysphagia is clinically important because it can affect the quality of life and prognosis of $\mathrm{ABI}$ patients.

In recent years, the noninvasive brain stimulation (NBS) methods of repetitive transcranial magnetic stimulation (rTMS) and transcranial direct current stimulation (tDCS) have been used for patients with $\mathrm{ABI}$ as a therapeutic neuromodulation tool (2). RTMS is a painless, noninvasive method that modulates cortical excitability. High-frequency rTMS facilitates cortical excitability, while low-frequency rTMS decreases cortical excitability of the stimulated hemisphere and increases cortical excitability of the nonstimulated hemisphere (3). TDCS is a non-invasive method used to modulate cortical excitability by applying direct current to the brain. During stimulation, the anode electrode causes enhancement of cortical excitability while the cathode electrode generates the opposite effect (4).

Several randomized controlled trials (RCTs) of NBS for dysphagia have been published in recent years (5-7). A systematic review of trials examining the efficacy and acceptability of NBS would be informative and useful for clinicians and researchers. Thus, the purpose of this study was to review the best available evidence of noninvasive brain stimulation for post- acquired brain injury dysphagia systematically, and to show the future direction of research in

Received for publication April 11, 2016 ; accepted May 31, 2016.

Address correspondence and reprint requests to Ryo Momosaki, MD, $\mathrm{PhD}, \mathrm{MPH}$, Department of Rehabilitation Medicine, The Jikei University School of Medicine, 3-25-8, Nishi-Shimbashi, Minato-Ku, Tokyo 1058461, Japan and Fax : +81-3-3431-1206. this field.

\section{MATERIALS AND METHODS}

Types of studies

We included only RCTs in this meta-analysis and excluded quasiRCTs and randomized controlled cross-over trials. Abstracts and non-English language publications were also excluded.

\section{Types of participants}

We included patients of any gender and older than 18 years of age with $\mathrm{ABI}$ of a non-degenerative nature. This included traumatic brain injury and stroke regardless of the duration of disease or severity of the disability. We excluded any condition of a progressive nature.

\section{Types of interventions and comparisons}

We included NBS (rTMS or tDCS) therapy alone or NBS plus rehabilitation. We excluded NBS plus peripheral electrical stimulation. The control interventions were sham treatment, sham treatment plus rehabilitation or no intervention. We excluded peripheral electrical stimulation.

\section{Outcomes}

We assessed outcomes at the time immediately after intervention. The primary outcomes were the Dysphagia Severity Rating Scales (Functional Oral Intake Scale : FOIS (8), Dysphagia Outcome Severity Scale : DOSS (9), Food Intake LEVEL Scale : FILS (10)). The secondary outcome was the penetration-aspiration scale : PAS (11) based on videofluoroscopy or videoendoscopic examination.

\section{Search strategy}

All relevant published studies were identified by searching the 
following databases : MEDLINE via STN (2004 to December 2015), EMBASE via STN (2004 to December 2015), CINAHL via EBSCO (to December 2015), and PEDro (to December 2015). All searches were completed in December 2015 (Appendix 1-4).

Appendix 1. MEDLINE (STN) search strategy

L1 : CRANIOCEREBRAL TRAUMA+NT/CT OR STROKE+NT/CT OR ?STROKE? OR (BRAIN? OR ?CRANIAL? OR ?CEREBR? OR ? CEPHAL? OR HEAD? OR NEUROSURG?) (3A) (INJUR? OR DAMAG? ORIMPAIR? OR HARM? OR LOSS? OR ?TRAUMA? OR ACCIDEN?) L2 : RTMS OR REPETITIVE?(2A)TRANSCRANIAL?(2A)MAGNETIC? (2A)STIMULAT? OR REPETITIVE?(2A)TMS

L3 : TDCS OR TRANSCRANIAL?(2A) (DIRECT?(2A)CURRENT? OR DC) (2A)STIMUL? OR TRANSCRANIAL?(2A)DCS

L4 : DEGLUTITION+NT/CT OR DEGLUTITION DISORDERS+NT/ CT OR DEGLUTITION? OR DYSPHAGI? OR SWALLOW?

L5 : RANDOMIZED CONTROLLED TRIAL/DT OR (RANDOM ALLOCATION+NT OR SINGLE-BLIND METHOD+NT OR DOUBLEBLIND METHOD+NT)/CT OR ((SINGL? OR DOUBLE? OR TREBL? OR TRIPL?) (W) (BLIND? OR MASK?) OR RANDOM? (3A) (?LOCAT? OR TRIAL? OR STUD? OR COMPAR? OR ASSIGN? OR DIVID?) OR RANDOMI?)/TI,AB

L6 : L2 OR L3 AND (L1 AND L4 AND L5)

L7 : (L6/HUMAN OR (L6 NOT ANIMALS+NT/CT)) AND PY>= 2004

Appendix 2. EMBASE (STN) search strategy

L1 : HEAD INJURY+NT/CT OR CEREBROVASCULAR ACCIDENT+ NT/CT OR STROKE PATIENT+NT/CT OR ?STROKE? OR (BRAIN? OR ?CRANIAL? OR ?CEREBR? OR ?CEPHAL? OR HEAD? OR NEUROSURG?) (3A) (INJUR? OR DAMAG? OR IMPAIR? OR HARM? OR LOSS? OR ?TRAUMA? OR ACCIDEN?)

L2 : RTMS OR REPETITIVE?(2A)TRANSCRANIAL?(2A)MAGNETIC? (2A)STIMULAT? OR REPETITIVE?(2A)TMS

L3 : TDCS OR TRANSCRANIAL?(2A) (DIRECT?(2A)CURRENT? OR DC) (2A)STIMUL? OR TRANSCRANIAL?(2A)DCS

L4 : SWALLOWING+NT/CT OR DYSPHAGIA+NT/CT OR DEGLUTITION? OR DYSPHAGI? OR SWALLOW?

L5 : (RANDOMIZED CONTROLLED TRIAL+NT OR RANDOMIZATION+NT OR SINGLE BLIND PROCEDURE+NT OR DOUBLE BLIND PROCEDURE+NT)/CT OR ((SINGL? OR DOUBLE? OR TREBL? OR TRIPL?) (W) (BLIND? OR MASK?) OR RANDOM?(3A) (?LOCAT? OR TRIAL? OR STUD? OR COMPAR? OR ASSIGN? OR DIVID?) OR RANDOMI?)/TI,AB

L6 : L2 OR L3 AND (L1 AND L4 AND L5)

L7 : (L6/HUMAN OR (L6 NOT ANIMALS+NT/CT)) AND PY > = 2004

Appendix 3. CINAHL (EBSCO) search strategy

S1 : HEAD INJURY + NT/CT OR CEREBROVASCULAR ACCIDENT+ NT/CT OR STROKE PATIENT+NT/CT OR ?STROKE? OR (BRAIN? OR ?CRANIAL? OR ?CEREBR? OR ?CEPHAL? OR HEAD? OR NEUROSURG?) (3A) (INJUR? OR DAMAG? OR IMPAIR? OR HARM? OR LOSS? OR ?TRAUMA? OR ACCIDEN?)

S2 : RTMS OR REPETITIVE?(2A)TRANSCRANIAL?(2A)MAGNETIC? (2A)STIMULAT? OR REPETITIVE?(2A)TMS

S3 : TDCS OR TRANSCRANIAL?(2A) (DIRECT?(2A)CURRENT? OR DC) (2A)STIMUL? OR TRANSCRANIAL?(2A)DCS

S4 : SWALLOWING+NT/CT OR DYSPHAGIA+NT/CT OR DEGLUTITION? OR DYSPHAGI? OR SWALLOW?

S5 : S2 OR S3 AND (S1 AND S4)

Appendix 4. PEDro search strategy

1. repetitive transcranial magnetic stimulation

2. transcranial direct current stimulation

\section{Selection of studies}

Two authors (RM and SK) independently reviewed all potential studies for inclusion against the eligibility criteria. They examined the title and abstract and, where necessary, the full text of studies to assess if they are eligible for inclusion. If they could not reach agreement by discussion, a third author $(\mathrm{NY})$ made the final decision about eligibility.

\section{Data extraction}

Two authors (RM and SK) used a standard form to extract study characteristics and outcome data from the studies independently. Discrepancies were checked against the original data. A third author (NY) made the final decision in case of disagreement. One author (RM) entered the data in Review Manager (RevMan) meta-analysis software Version 5.3. (Copenhagen : The Nordic Cochrane Centre, The Cochrane Collaboration, 2011).

\section{Assessment of risk of bias}

We assessed the methodological quality of selected studies as described in the Cochrane Review Groups (12). We created a Risk of bias table and included description and judgment (low risk of bias, high risk of bias, or unclear risk of bias) for the following domains for each of the included studies : 1 . random sequence generation; 2. allocation concealment ; 3 . blinding of participants and personnel ; 4 . blinding of outcome assessment ; 5 . incomplete outcome data ; 6 . selective reporting; 7 . other sources of bias. Two review authors independently performed quality assessment. Any disagreement between authors arising at any stage was resolved through discussion or through a third author.

\section{Statistical analysis}

For all outcomes related to continuous data, we calculated a pooled estimate of the standardized mean difference (SMD) with 95\% confidence interval (CI). We used a generic inverse variance method and random effects model to combine individual results. The threshold for significance was set at $\mathrm{p}<0.05$. For all statistical comparisons we used RevMan 5.3. We used the $\mathrm{I}^{2}$ statistic to assess heterogeneity. $I^{2}>50 \%$ was considered to reflect substantial heterogeneity (13). We used the funnel plot method for assessment of reporting biases only when there were at least 10 studies included in the meta-analysis (14). Statistical analysis was conducted by dividing NBS into rTMS or tDCS. We used the criteria of the Grading of Recommendations Assessment, Development and Evaluation (GRADE) to evaluate the quality of the evidence by outcome. This study was prospectively registered with the PROSPERO database of systematic reviews (CRD42015032269)

\section{RESULTS}

\section{Results of the search}

We identified 291 records through the searches after removal of duplicates. After screening the titles and abstracts, we excluded 163 records mainly because the studies were animal studies, abstracts only and non-English language publications. After further assessment, 8 studies ( 5 rTMS and 3 tDCS studies) were considered to meet the review inclusion criteria (Figure 1).

\section{Characteristics of patients}

The meta-analysis was based on 8 studies (15-22) that included 188 patients. Table 1 provides a comprehensive summary of the 8 studies. The mean age of participants ranged from 57 to 75 years. The proportion of women was $27 \%$ to $65 \%$ among these trials. Most studies did not include information on ABI severity or activities of daily living. All studies included data on stroke (infarction or 


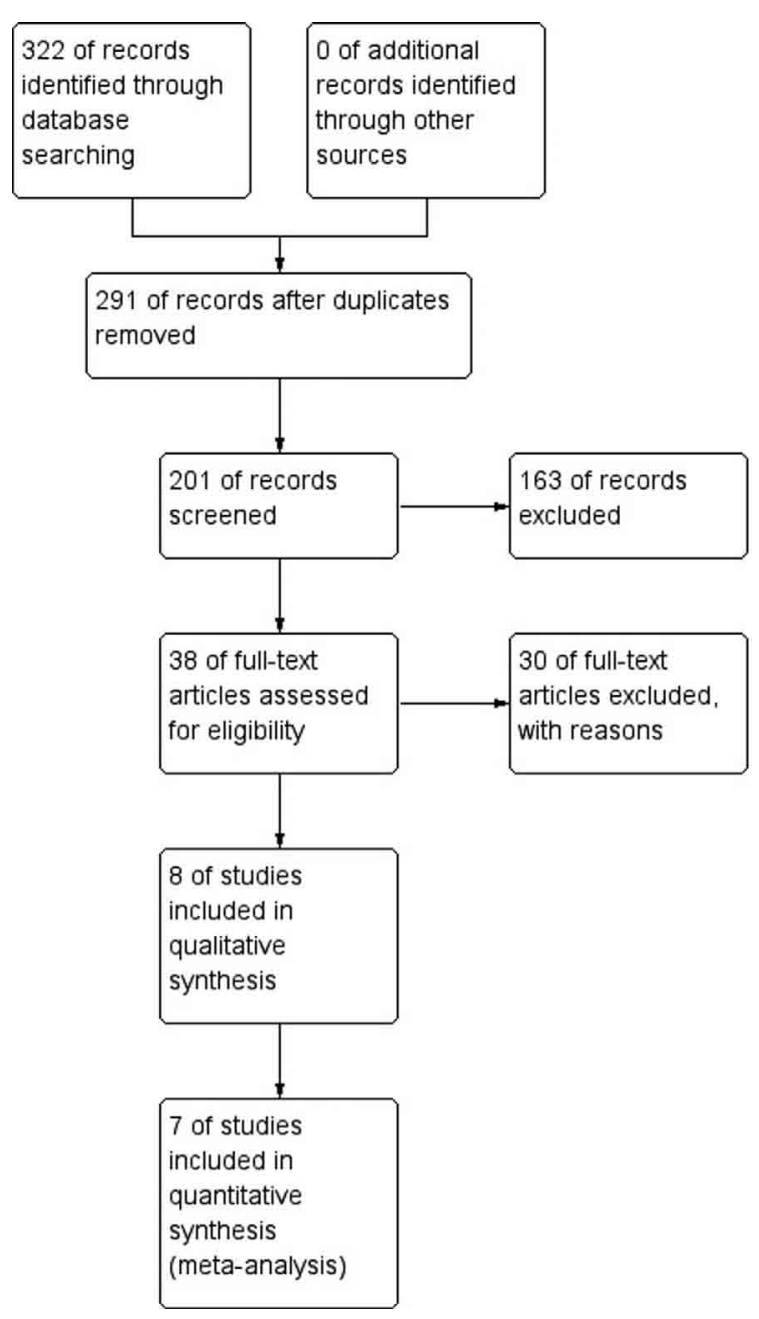

Figure 1 : Flowchart of study selection

hemorrhage) except for one study, which included patients with TBI (2 participants) (17). Almost all patients had unilateral cerebral hemisphere damage. However, two studies included patients with infratentorial lesions $(16,22)$. The severity of dysphagia ranged between moderate and severe. Almost all trials included patients with acute to sub-acute phase stroke (up to 3 months from onset), although one study included chronic patients (more than six months from onset) (22). The follow-up period ranged from 1 to 90 days after the end of intervention.

\section{Interventions}

Trials were divided into three categories based on the intervention : 1 . rTMS compared with sham rTMS $(15,16,18) ; 2$. rTMS with swallowing exercise compared with sham rTMS with swallowing exercise $(17,19)$; and 3 . tDCS with swallowing exercise compared with sham $\mathrm{tDCS}$ with swallowing exercise (20-22). The frequency of rTMS ranged from 1 to $5 \mathrm{~Hz}$. TDCS was applied using 1 or $2 \mathrm{~mA}$. Stimulation was applied to the ipsilateral, contralateral or bilateral motor area. The treatment protocols included 5-10 sessions, with each including the application of stimulation for 10-30 $\min /$ day.

\section{Outcome measures}

Four trials $(15,16,20,22)$ used DOSS and 3 trials $(17-19)$ used PAS for assessment of outcome. Other reported outcome measures included Functional Dysphagia Scale : FDS (23), American Speech-Language-Hearing Association National Outcome Measurement System swallowing scale : ASHS NOMS swallowing scale (24), Videofluoroscopic Dysphagia Scale : VDS (25) and Pharyngeal Transit Time : PTT (26). All trials included the outcomes at the end of treatment period. We excluded one trial from qualitative synthesis (21) because it did not provide appropriate outcome data.

\section{Risk of bias}

Next, we analyzed the risk of bias in characteristics of included studies. For this purpose, we drew risk of bias summary based on each risk of bias item presented as percentages across all included studies (Figure 2). The method of allocation concealment was not described or not described in sufficient detail to allow a definite judgment in 6 studies. Because complete blinding to sham rTMS is difficult in such studies, we judged that all studies using rTMS involved high risk of bias for blinding of participants and personnel. All of the included studies were low risk of bias for incomplete outcome data. Some studies had a few missing outcome data, although these balanced in numbers across the intervention groups, with similar reasons for missing data across groups. There was insufficient information to make judgment on selective reporting. One study completed trial registration before the study and thus conformed low risk of selective reporting (20). The number of studies was insufficient to employ funnel plot analysis.

Table 1: Details of included studies

\begin{tabular}{|c|c|c|c|c|c|c|}
\hline Study & Participants & $\begin{array}{l}\text { Time from } \\
\text { onset (days) }\end{array}$ & Stimulation type & $\begin{array}{l}\text { Target cortex } \\
\text { of stimulation }\end{array}$ & Stimulation program & Outcome measure \\
\hline Khedr et al. 2009 & 26 Stroke & $5-10$ & rTMS $3 \mathrm{~Hz}$ & Ipsilateral & $10 \mathrm{~min} /$ day, 5 session & DOSS \\
\hline Khedr et al. 2010 & 22 Stroke & $1-90$ & rTMS $3 \mathrm{~Hz}$ & Bilateral & $10 \mathrm{~min} /$ day, 5 session & DOSS \\
\hline Kim et al. 2011 & 28 Stroke 2 TBI & $1-90$ & $\begin{array}{l}\text { rTMS } 1 \text { or } 3 \mathrm{~Hz} \\
+ \text { exercise }\end{array}$ & $\begin{array}{l}\text { Ipsilateral or } \\
\text { Contralateral }\end{array}$ & $20 \mathrm{~min} /$ day, 10 session & $\begin{array}{c}\text { PAS, FDS, ASHS } \\
\text { NOMS }\end{array}$ \\
\hline Park et al. 2013 & 18 Stroke & $1-90$ & rTMS 5Hz & Contralateral & $10 \mathrm{~min} /$ day, 10 session & PAS VDS \\
\hline Lim et al. 2014 & 40 Stroke & $1-90$ & rTMS $1 \mathrm{~Hz}+$ exercise & Contralateral & $20 \mathrm{~min} /$ day, 10 session & $\begin{array}{l}\text { PAS, FDS, ASHS } \\
\text { NOMS, PTT }\end{array}$ \\
\hline Kumar et al. 2010 & 14 Stroke & & tDCS $2 \mathrm{~mA}+$ exercise & Contralateral & $30 \mathrm{~min} /$ day, 5 session & DOSS \\
\hline Yang et al. 2012 & 18 Stroke & $1-60$ & tDCS $1 \mathrm{~mA}+$ exercise & Ipsilateral & $20 \mathrm{~min} /$ day, 10 session & FDS \\
\hline Sigematsu et al. 2013 & 20 Stroke & $30-300$ & tDCS 1mA+exercise & Ipsilateral & $20 \mathrm{~min} /$ day, 10 session & DOSS \\
\hline
\end{tabular}

DOSS : Dysphagia Outcome Severity Scale ; PAS : Penetration Aspiration Scale ; FDS : Functional Dysphagia Scale ; ASHS NOMS : American Speech-Language-Hearing Association National Outcome Measurement System swallowing scale ; VDS : Videofluoroscopic Dysphagia Scale : PTT : Pharyngeal Transit Time ; rTMS : repetitive transcranial magnetic stimulation ; tDCS : transcranial direct current stimulation ; TBI : traumatic brain injury. 


\begin{tabular}{|c|c|c|c|c|c|c|c|}
\hline Study & $\begin{array}{l}\text { Random } \\
\text { sequence } \\
\text { generation }\end{array}$ & $\begin{array}{l}\text { Allocation } \\
\text { concealment }\end{array}$ & $\begin{array}{l}\text { Blinding of } \\
\text { participants } \\
\text { and personnel }\end{array}$ & $\begin{array}{l}\text { Blinding of } \\
\text { outcome } \\
\text { assessment }\end{array}$ & $\begin{array}{l}\text { Incomplete } \\
\text { outcome data }\end{array}$ & $\begin{array}{l}\text { Selective } \\
\text { reporting }\end{array}$ & Other bias \\
\hline $\begin{array}{l}\text { Khedr etal } \\
2009\end{array}$ & $\triangle$ & & $x$ & & & $\triangle$ & $\Delta$ \\
\hline $\begin{array}{l}\text { Khedr etal } \\
2010\end{array}$ & $\Delta$ & 0 & $x$ & & & $\Delta$ & $\Delta$ \\
\hline $\begin{array}{l}\text { Kimet al } \\
2011\end{array}$ & $\triangle$ & $\triangle$ & $x$ & $x$ & & $\triangle$ & $\triangle$ \\
\hline $\begin{array}{l}\text { Park et al } \\
2013\end{array}$ & 0 & 0 & $x$ & & & $\Delta$ & $\Delta$ \\
\hline $\begin{array}{l}\text { Limet al } \\
2014\end{array}$ & 0 & $\triangle$ & $x$ & & & $\triangle$ & $\triangle$ \\
\hline $\begin{array}{l}\text { Kumar et al } \\
2010 \\
\end{array}$ & $\triangle$ & $\triangle$ & & & & 0 & $\Delta$ \\
\hline $\begin{array}{l}\text { Yang etal } \\
2012\end{array}$ & $\triangle$ & $\triangle$ & & & ) & $\triangle$ & $\triangle$ \\
\hline $\begin{array}{l}\text { Sigematsuet al } \\
2013\end{array}$ & $\triangle$ & $\triangle$ & 0 & & 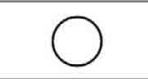 & $\Delta$ & $\Delta$ \\
\hline
\end{tabular}

Figure 2 : Risk of bias summary

Review authors' judgments about each risk of bias item for each included study. Circle indicates low risk of bias, cross high risk of bias, while triangle indicates unclear risk of bias.

\section{Effects of interventions}

Comparison : rTMS versus control

With regard to the primary outcome, two trials with 48 participants recorded DOSS immediately after intervention. Meta-analysis showed that rTMS intervention was associated with a significant improvement in DOSS (SMD : 2.95, 95\% CI : 1.02-4.88, P=0.003). However, there was statistically significant heterogeneity between the trials $\left(I^{2}=77 \%\right)$ (Figure 3 ).

Three trials that included 88 patients recorded PAS immediately after the intervention. Meta-analysis showed that rTMS intervention was associated with a significant improvement in PAS (SMD : $0.77,95 \% \mathrm{CI}: 0.33-1.21, \mathrm{P}=0.0006)$. There was no statistically significant heterogeneity between the trials $\left(I^{2}=0 \%\right.$ ) (Figure 4$)$.

\section{Comparison : $t D C S$ versus control}

With regard to the primary outcome, two trials with 34 participants recorded DOSS immediately after intervention. Meta-analysis showed that $\mathrm{tDCS}$ intervention was associated with a significant improvement in DOSS (SMD : 1.20, 95\% CI : 0.45-1.95, $\mathrm{P}=0.002$ ). There was no statistically significant heterogeneity between the trials $\left(I^{2}=0 \%\right)$ (Figure 5$)$. There were no studies concerning tDCS for PAS.

See : Summary of findings for the main comparison NIBS vs. control (Figure 6).

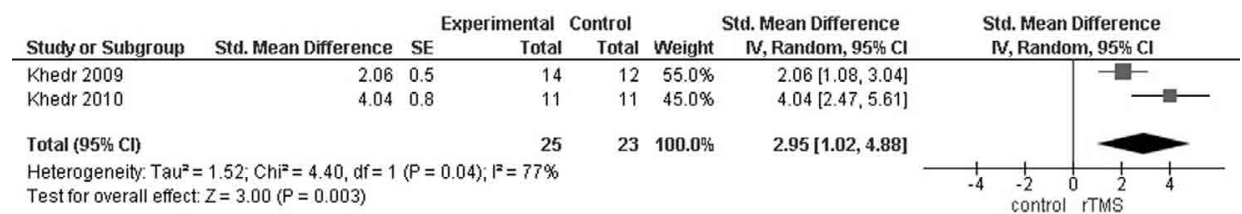

Figure 3 : Forest plot of the association of repetitive transcranial magnetic stimulation with dysphagia outcome and severity scale CI, confidence interval ; Std, standard ; SE, standard error.

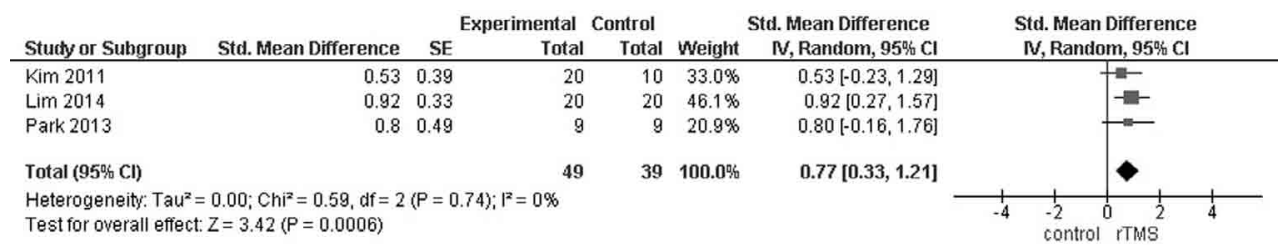

Figure 4 : Forest plot of the association of repetitive transcranial magnetic stimulation with penetration-aspiration scale CI, confidence interval ; Std, standard ; SE, standard error. 


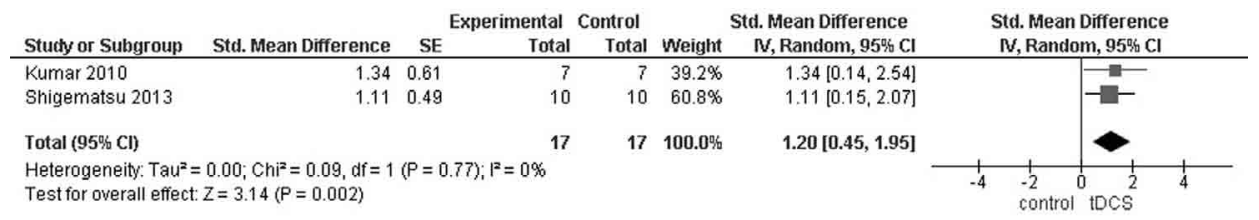

Figure 5 : Forest plot of the association of transcranial direct current stimulation with dysphagia outcome and severity scale $\mathrm{CI}$, confidence interval ; Std, standard ; SE, standard error.

Noninvasive brain stimulation compared with control intervention for dysphagia

Patient or population: acquired brain injury with dysphagia; Intervention: Noninvasive brain stimulation; Comparison: control intervention

\begin{tabular}{|c|c|c|c|c|}
\hline Outcomes & $\begin{array}{l}\text { Relative effect } \\
\qquad(95 \% \mathrm{Cl})\end{array}$ & $\begin{array}{c}\text { No of Participants } \\
\text { (studies) }\end{array}$ & $\begin{array}{l}\text { Quality of the evidence } \\
\text { (GRADE) }\end{array}$ & Comments \\
\hline $\begin{array}{l}\text { Dysphagia Severity } \\
\text { Rating Scale (rTMS) }\end{array}$ & $\begin{array}{c}\text { SMD } 2.95 \\
\text { (1.02 to } 4.88)\end{array}$ & $\begin{array}{l}48 \text { participants } \\
\text { ( } 2 \text { studies) }\end{array}$ & $\oplus \Theta \Theta \Theta$ very low $\mathbf{w}^{1,2,3,4}$ & \\
\hline $\begin{array}{l}\text { Dysphagia Severity } \\
\text { Rating Scale (tDCS) }\end{array}$ & $\begin{array}{l}\text { SMD } 1.20 \\
(0.45 \text { to } 1.95)\end{array}$ & $\begin{array}{l}88 \text { participants } \\
\text { ( } 3 \text { studies) }\end{array}$ & $\oplus \oplus \Theta \Theta$ low $^{1,3}$ & \\
\hline $\begin{array}{l}\text { Penetration-aspiration } \\
\text { scale (rTMS) }\end{array}$ & $\begin{array}{c}\text { SMD 0.77 } \\
(0.33 \text { to } 1.21)\end{array}$ & $\begin{array}{l}34 \text { participants } \\
\text { ( } 2 \text { studies) }\end{array}$ & $\oplus \Theta \Theta \Theta$ very low $w^{1,4,5}$ & \\
\hline
\end{tabular}

\begin{tabular}{l} 
Penetration-aspiration \\
Scale (tDCS) \\
\hline rTMS: repetitive transcranial magnetic stimulation; tDCS: transcranial direct current stimulation; Cl: Confidence interval; SMD: Standardized mean difference \\
\hline GRADE Working Group grades of evidence \\
Low quality: Further research is very likely to have an important impact on our confidence in the estimate of effect and is likely to change the estimate. \\
Very low quality: We are very uncertain about the estimate. \\
\hline 1 Small total population size. \\
${ }^{2}$ Statistical heterogeneity was moderate to high. \\
${ }^{3}$ Random sequence generation was unclear. \\
${ }^{4}$ Unclear blinding of participants and personnel. \\
${ }^{5}$ Small effect size. \\
${ }^{6}$ Data were not pooled.
\end{tabular}

Figure 6 : Summary of findings for the main comparison.

\section{DISCUSSION}

This review focused on evaluating the best available evidence of rTMS or tDCS versus control for improvement of swallowing function after ABI. We included 8 trials that included 188 patients. Two studies of 48 patients using rTMS and two studies of 34 patients using tDCS examined the effects on the primary outcome measure selected in our study ; the Dysphagia Severity Rating Scale after ABI. We found evidence of a favorable effect of rTMS and tDCS on DOSS at the end of the intervention. Three studies of 88 participants assessed the effects of rTMS on PAS at the end of intervention and there was significant evidence for the effect at the end of the intervention.

\section{Overall completeness and applicability of evidence}

The results of the review were limited by the following factors. First, the stimulation protocol (frequency, pulses, target of stimulation) varied across studies. Therefore, we were unable to select the most suitable rTMS and tDCS protocol. Second, most participants suffered from supratentorial stroke in acute or subacute phage. Thus, the results may be of limited value to patients with infratentorial lesion, chronic stroke or traumatic brain injury. Third, the longest follow-up period was 3 months. Thus, the long-term effect of the interventions remains unclear at this stage. Forth, we did not cover trials that are still unpublished or in press, published in non-English languages or published in abstracts only. The above limitations/study protocol point to some potential bias in the review process. Fifth, the total number of studies was small. Therefore, it may be too early to perform meta-analysis for this topic. However, the aim of this study was to review the best available evidence to show the future direction of research. If the number of studies is small, it is still meaningful to combine the best available evidence. Almost all Cochrane review groups follow such a standardized editorial and publishing policy. We followed a similar policy. Furthermore, we registered the analysis protocol to combine the best available evidence. The policy safeguards against changing the analysis and reporting depending on the results (that is breaking the protocol), and introducing selective reporting bias. For these reasons, we conducted meta-analysis according to our registered protocol.

\section{Quality of evidence}

In general, the quality of reporting was poor. Most trials reported random assignment but the methods of randomization were not described in full detail. Compared with rTMS and tDCS, sham rTMS is harder to perform than sham tDCS with regard to blinding of participants and personnel. Although all trials used sham rTMS in the control group, the success of blinding was not recorded. There was large heterogeneity between the studies, particularly for rTMS. The potential problem associated with this heterogeneity relates to the affected brain region ; supra- and infratentorial areas. The sample size was small, ranging from 14 to 40 patients, and the number of studies was not sufficient to assess publication bias. Thus, we call for large RCTs to verify the efficacy of rTMS and tDCS for dysphagia.

\section{Conclusions}

Low quality evidence suggests the efficacy of rTMS and tDCS in improving dysphagia after ABI. This evidence, however, needs to be substantiated and confirmed. Routine use of NBS in patients 
with $\mathrm{ABI}$ should be avoided until its efficacy is verified in highquality, large-scale RCTs.

There are some ongoing clinical trials in this field (tDCS : ISRCTN97286108, rTMS : NCT02090231). Future studies with large sample size that include patients with infra- and supra-tentorial lesions and acute and chronic stroke are needed to validate the beneficial effects of NBS for dysphagia. Particularly, the most suitable rTMS and tDCS protocol should be defined and functional outcome measured at long-term follow-up should be used as the primary outcome.

\section{CONFLICTS OF INTEREST}

The authors declare that they have no conflict of interest.

\section{ACKNOWLEDGEMENTS}

This study was funded by a grant from the Japan Society for Promotion of Science KAKENHI (grant number 70439800).

\section{REFERENCES}

1. Feigin VL, Barker-Collo S, Krishnamurthi R, Theadom A Starkey N : Epidemiology of ischaemic stroke and traumatic brain injury. Best Pract Res Clin Anaesthesiol 24 : 485-494, 2010

2. Williams JA, Imamura M, Fregni F : Updates on the use of noninvasive brain stimulation in physical and rehabilitation medicine. J Rehabil Med 41 : 305-311, 2009

3. Hsu WY, Cheng CH, Liao KK, Lee IH, Lin YY : Effects of repetitive transcranial magnetic stimulation on motor functions in patients with stroke : a meta-analysis. Stroke $43: 1849$ 1857,2012

4. Jacobson L, Koslowsky M, Lavidor M : tDCS polarity effects in motor and cognitive domains : a meta-analytical review. Exp Brain Res $216: 1-10,2011$

5. Rofes L, Vilardell N, Clavé P : Post-stroke dysphagia : progress at last. Neurogastroenterol Motil 25 : 278-282, 2013

6. Barwood CH, Murdoch BE : rTMS as a treatment for neurogenic communication and swallowing disorders. Acta Neurol Scand 127 : 77-91, 2013

7. Feng WW, Bowden MG, Kautz S : Review of transcranial direct current stimulation in poststroke recovery. Top Stroke Rehabil 20 : 68-77, 2013

8. Crary MA, Mann GD, Groher ME : Initial psychometric assessment of a functionaloral intake scale for dysphagia in stroke patients. Arch Phys Med Rehabil 86 : 1516-1520, 2015

9. O'Neil KH, Purdy M, Falk J, Gallo L: The Dysphagia Outcome and Severity Scale. Dysphagia $14: 139-145,1999$

10. Kunieda K, Ohno T, Fujishima I, Hojo K, Morita T : Reliability and validity of a tool to measure the severity of dysphagia : the
Food Intake LEVEL Scale. J Pain Symptom Manage 46 : 201206, 2013

11. Rosenbek JC, Robbins JA, Roecker EB, Coyle JL, Wood JL : A penetration-aspiration scale. Dysphagia $11: 93-98,1996$

12. Lundh A, Gøtzsche PC : Recommendations by Cochrane Review Groups for assessment of the risk of bias in studies. BMC Med Res Methodol $8: 22,2008$

13. Higgins JP, Thompson SG, Deeks JJ, Altman DG : Measuring inconsistency in meta-analyses. Br Med J 327 : 557-560, 2003

14. Souza JP, Pileggi C, Cecatti JG : Assessment of funnel plot asymmetry andpublication bias in reproductive health metaanalyses : an analytic survey. Reprod Health $4: 3,2007$

15. Khedr EM, Abo-Elfetoh N, Rothwell JC : Treatment of poststroke dysphagia with repetitive transcranial magnetic stimulation. Acta Neurol Scand 119 : 155-161, 2009

16. Khedr EM, Abo-Elfetoh N : Therapeutic role of rTMS on recovery of dysphagia in patients with lateral medullary syndrome and brainstem infarction. J Neurol Neurosurg Psychiatry 81 : 495-459, 2010

17. Kim L, Chun MH, Kim BR, Lee SJ : Effect of repetitive transcranial magnetic stimulation on patients with brain injury and dysphagia. Ann Rehabil Med 35 : 765-771, 2011

18. Park JW, Oh JC, Lee JW, Yeo JS, Ryu KH : The effect of $5 \mathrm{~Hz}$ high-frequency rTMS over contralesional pharyngeal motor cortex in post-stroke oropharyngeal dysphagia : a randomized controlled study. Neurogastroenterol Motil 25 : 324-e250, 2013

19. Lim KB, Lee HJ, Yoo J, Kwon YG : Effect of Low-Frequency rTMS and NMES on Subacute Unilateral Hemispheric Stroke With Dysphagia. Ann Rehabil Med 38 : 592-602, 2014

20. Kumar S, Wagner CW, Frayne C, Zhu L, Selim M, Feng W, Schlaug G : Noninvasive brain stimulation may improve strokerelated dysphagia : a pilot study. Stroke $42: 1035-1040,2011$

21. Yang EJ, Baek SR, Shin J, Lim JY, Jang HJ, Kim YK, Paik NJ : Effects of transcranial direct current stimulation (tDCS) on post-stroke dysphagia. Restor Neurol Neurosci 30 : 303-311, 2012

22. Shigematsu T, Fujishima I, Ohno K : Transcranial direct current stimulation improves swallowing function in stroke patients. Neurorehabil Neural Repair 27 :363-369, 2013

23. Han TR, Paik NJ, Park JW : Quantifying swallowing function after stroke : A functional dysphagia scale based on videofluoroscopic studies. Arch Phys Med Rehabil 82 : 677-682, 2001

24. Kim J, Oh BM, Kim JY, Lee GJ, Lee SA, Han TR : Validation of the videofluoroscopic dysphagia scale in various etiologies. Dysphagia 29 : 438-443, 2014

25. Kim DH, Choi KH, Kim HM, Koo JH, Kim BR, Kim TW, Ryu JS, Im S, Choi IS, Pyun SB, Park JW, Kang JY, Yang HS : Interrater Reliability of Videofluoroscopic Dysphagia Scale. Ann Rehabil Med 36 : 791-796, 2012

26. Hamlet SL, Muz J, Patterson R, Jones L: Pharyngeal transit time : assessment with videofluoroscopic and scintigraphic techniques. Dysphagia $4:$ 4-7, 1989 